\title{
Ferromagnetic Resonance Force Microscopy: Spectroscopy on the Nano-Scale
}

\author{
S. P. White ${ }^{1}$, P. C. Hammel ${ }^{1}$ \\ ${ }^{1}$ Department of Physics, The Ohio State University, Columbus, OH 43210
}

Magnetic heterostructures are commonly employed in the growing field of spintronics which utilizes spin transport in materials systems that combine ferromagnetic (FM) materials and normal, or non-magnetic, metals (NM). However, an understanding of how spin is accumulated and transported across these interfaces between two dissimilar materials is needed in order to improve and implement nano-scale structures for future spintronic applications. An equally important question is how magnetic properties such as the local internal field vary spatially in devices. Simulations have shown that variations in the anisotropy fields in a permalloy (Py) film can have large impacts on the linewidth measured by ferromagnetic resonance (FMR), determined by the length scales over which the anisotropy varies [1]. With the linewidth being an important figure of merit for FM devices, this fact motivates the need for a tool that can study dynamical magnetic properties on relevant length scales.

Conventional FMR is a powerful technique for studying the internal fields in a FM. However, due its use of global excitation methods combined with detection that is insufficiently sensitive to measure small volumes, it cannot map varying internal fields with the needed spatial resolution. Ferromagnetic resonance force microscopy (FMRFM), a powerful tool for microscopic study of magnetic properties in FM films, addresses these issues and provides a strong complement to conventional FMR. This technique uses a micromagnetic particle attached near the end of a cantilever that interacts with the nearby volume of a FM. Due to the strong spin-spin interactions in a FM, the excitations of a FM are collective modes whose typically large spatial extent limits spatial resolution.

Localized mode FMRFM improves the resolution to the micro- or nano-scale through the use of a micromagnet with a large moment that localizes spin wave modes into a small volume beneath the micromagnetic tip [2]. Magnetizing the FM film out of plane, and then using a magnetic probe anti-aligned to the applied field reduces the internal field in the FM below the particle. Figure 1 illustrates how the dipole field of the magnetic particle on the cantilever creates a localizing field well directly beneath the particle in the FM film. By studying the spectrum of spin waves trapped in the well, static properties such as the local internal field of the FM are measured, as well as dynamic properties such as the transport of spin angular momentum to the surrounding quiescent material [3]. This technique allows the investigation of spatially varying magnetic properties in spatially extended pristine films of conducting and insulating FM materials. The spin current passes exclusively through field-defined interfaces, avoiding issues such as material heterointerfaces and sample variation, and allows in-operando tuning of the properties of the well in contrast to the immutability of lithographically defined structures.

We are studying interfaces that are defined by spatially varying internal fields rather than dipolar fields of a tip. We have imaged a sharp discontinuity in the internal field that separates two regions of a continuous Py thin film [4]. Such interfaces provide another valuable tool for studying magnetization dynamics at interfaces. These two regions are distinguished by irradiating half of the Py film with helium ions as shown in Figure 1. This increases the saturation magnetization in the irradiated area resulting in an internal field step of approximately 400 Gauss. We imaged the interface by means of localized mode FMRFM. Modes localized by the probe field have a resonance condition set by the excitation frequency 
of microwaves applied by a nearby stripline and the local internal field of the Py thin film. Due to this sensitivity to the local internal field, the value of the externally applied field, $H_{0}$, at which the mode resonates, is found to vary strongly with both proximity to the interface, as well as the depth of the well relative to the step height. Figure 2 illustrates three different cases: the depth of the dipole field well is (a) smaller than, (b) on the same order as, and (c) larger than the variation in the demagnetizing field between the two regions. The depth of the field well created by the magnetic particle is controlled by changing the probe-sample separation, $\mathrm{z}$. We used micromagnetic modeling to simulate and analyze the evolution of the mode shape and resonant field. The black squares in Figure 2 represent resonant fields extracted from simulations performed using the parameters applied in the experiment, and these agree well with the experimental data. We find that the interface can be detected with $\approx 100 \mathrm{~nm}$ resolution on the irradiated (IR) side, and $\approx 500 \mathrm{~nm}$ on the non-irradiated (NIR) side, the latter being affected by mode pinning at the interface.

We are expanding this technique to measure samples with two dimensional ion irradiated patterns. These measurements will focus on magnetization dynamic in local modes, the interactions between two spatially separated modes, and the coupling of localized modes to imposed spin currents [5].

\section{References:}

[1] R.D. McMichael and D.J. Twisselmann, Phys. Rev. Lett. 90 (2003), p. 227601

[2] Inhee Lee et al, Nature 466 (2010), p. 845

[3] Rohan Adur et al, Phys. Rev. Lett. 113 (2014), p. 176601

[4] Chunhui Du et al, Phys. Rev. B 92 (2015), p. 214413

[5] The authors acknowledge funding from the US Department of Energy, Award No. DE-FG0203ER46054.

Figure 1. Schematic of localized mode FMRFM on a Py film. Internal fields are represented in the graph above the sample showing the combination of externally applied field, $H_{0}$, demagnetizing field of the irradiated region (IR)/non-irradiated region (NIR), $4 \pi M_{I R / N I R}$, and parabolic dipolar field from the particle. The profile of a localized

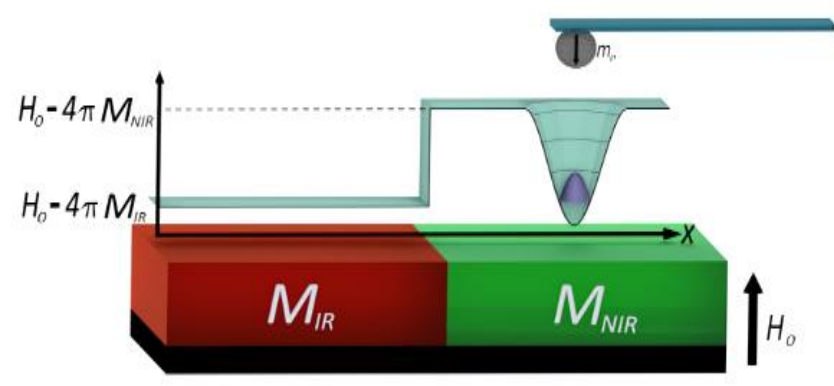
mode is shown within the field well.
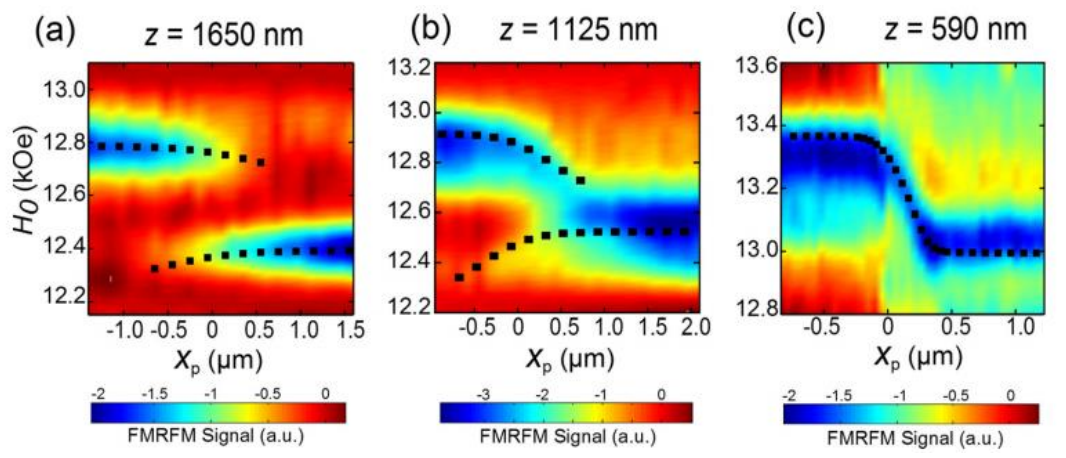

Figure 2. Two dimensional FMRFM images of applied external field versus lateral probe position for three probesample separations, $z$. Black squares represent resonant fields of localized modes obtained from micromagnetic simulations (from Ref. [4]). 